\title{
Application of SBAS Technique Combined with BP Neural Network in the Settlement of the Yinxi Industrial Park in Baiyin
}

\author{
Hui Zhang ${ }^{1}$, Xinghai Dang ${ }^{1}$, , Liqi Jia ${ }^{2}$, Jianyun Zhao ${ }^{3}$, Ming Lu ${ }^{1}$ \\ ${ }^{1}$ School of Civil Engineering, Lanzhou University of Technology, Lanzhou, China \\ ${ }^{2}$ School of Design Arts, Lanzhou University of Technology, Lanzhou, China \\ ${ }^{3}$ Department of Geologic Engineering, Qinghai University, Xining, China \\ Email address: \\ 1027042853@qq.com (Hui Zhang), 40288878@qq.com (Xinghai Dang) \\ ${ }^{*}$ Corresponding author
}

\section{To cite this article:}

Hui Zhang, Xinghai Dang, Liqi Jia, Jianyun Zhao, Ming Lu. Application of SBAS Technique Combined with BP Neural Network in the Settlement of the Yinxi Industrial Park in Baiyin. American Journal of Civil Engineering. Vol. 9, No. 5, 2021, pp. 167-172. doi: 10.11648/j.ajce.20210905.13

Received: September 29, 2021; Accepted: October 20, 2021; Published: October 29, 2021

\begin{abstract}
In recent years, due to the obvious ground settlement and other phenomena of the Yinxi Industrial Park in Baiyin, it has brought many hidden dangers to the local development, it is of great practical significance to monitor the deformation of the area for a long time series. The ground deformation field of Yinxi Industrial Park from June 2018 to April 2021 was obtained by processing Sentinel-1A data using SBAS technology, and the high coherence point D1 was predicted and analyzed by BP neural network. The results show that subsidence occurs in several places in the Yinxi Industrial Park, and the average annual subsidence rate ranges from $-19.28 \mathrm{~mm}$ to $5.08 \mathrm{~mm}$, the areas of severe settlement have a clear geographical distribution, mainly concentrated in road and building areas, other areas have a more stable ground base; the mean square error in the BP neural network prediction result is $2.56 \mathrm{~mm}$, and the average relative error is $6.06 \%$, which is a high prediction accuracy. The predicted cumulative settlement value at point D1 in 2023 is $45 \mathrm{~mm}$, and there is a tendency for the settlement to intensify. The prediction results are of great significance for the early identification and prevention of ground settlement in the study area.
\end{abstract}

Keywords: SBAS Technology, Land Subsidence, BP Neural Network, Yinxi Industrial Park

\section{Introduction}

Ground settlement is a common geological problem in social and economic development [1-2], mainly caused by natural and man-made factors that lead to local or regional subsidence phenomena, usually occurring as a result of foundation instability, building damage and other geological disasters, and is one of the important obstacles to the health and sustainable development of the region [3]. Yinxi Industrial Park is located in Baiyin City, Gansu Province. In recent years, with the accelerated urbanisation process, many factors such as the construction of high-rise buildings and highways have led to the subsidence of the ground in many places, and it is particularly important to monitor and analyse the subsidence in the area in order to minimize the safety hazards brought by the subsidence to the local people.

Traditional ground measurement means are mainly based on level measurement, which is not able to meet the practical needs because of the small monitoring range and high cost, although the accuracy is high [4-5]. Synthetic aperture radar interferometry (InSAR) and Differential interferometric synthetic aperture radar (D-InSAR), as a rapidly developing remote sensing technique, can obtain surface deformation information through SAR phase information, which can achieve centimeter-level monitoring accuracy and greatly improve monitoring efficiency, but is severely affected by spatial and temporal decoherence [6]. Zhao Fumeng et al. 
used SBAS technology based on sentinel data for early identification of geological hazards in the Ghazi Valley of the China-Pakistan Highway and extracted regional deformation information of geological hazards [7], and Pan Guangyong et al. applied SBAS technology to mine subsidence monitoring and effectively obtained the annual average subsidence rate and cumulative subsidence in the mine area [8]. These studies show that it is feasible to apply SBAS technology to ground subsidence monitoring and identification on a large scale; however, there are fewer studies that combine different SBAS technologies with BP neural network models.

In this paper, we use SBAS technology to process and analyze 34 scenes of sentinel-1A data during the period from July 2018 to November 2021 in Yinxi Industrial Park, and predict the settlement points in the study area by BP neural network, in order to provide data support for early subsidence hazard identification and healthy and sustainable development in Yinxi Industrial Park.

\section{Study Area Overview and Experimental Data}

\subsection{Overview of the Study Area}

Yinxi Industrial Park is located in the southwest of Baiyin District, Baiyin City, Gansu Province, as shown in Figure 1, with an area of about 14.88 square kilometres, and is an important part of Baiyin Industrial Zone, including the West District Economic Development Zone and Baiyin District Small and Medium-sized Enterprise Park. In recent years, the area has been plagued by problems such as road surface collapse and wall cracking.

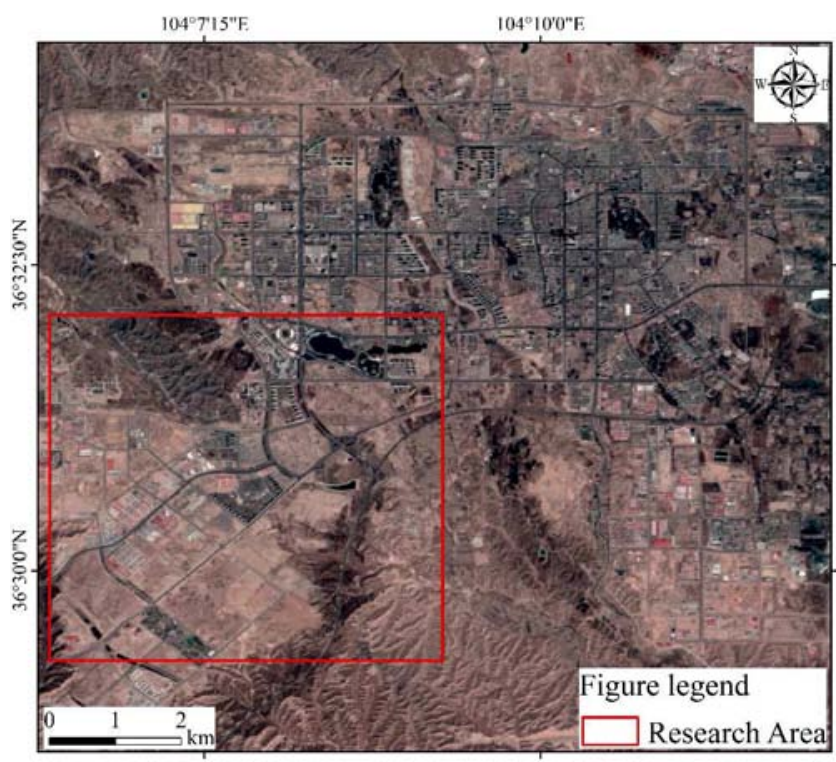

Figure 1. Study area.

\subsection{Experimental Data}

The Sentinel-1 radar satellite selected in this paper has a good penetrating capability C-band with a revisit period of 12 days, which enables long-term and frequent monitoring [9-10], (data from ESA official website: http://www.esa.int/), and the interferometric mode and polarization mode are wide interferometric mode (IW) and isotropic polarization (VV), respectively. The time span is June 2018-April 2021, and the orbit information is corrected using the corresponding POD precision orbit ephemeris data. The image data parameters are shown in Table 1 . The $30 \mathrm{~m}$ resolution DEM used with the interference topography phase removed is derived from: http:/gdex.cr.usgs.gov/gdex/.

Table 1. Parameters of Sentinel-1 data.

\begin{tabular}{ll}
\hline Satellite Model & Sentinel-1A \\
\hline Orbital Direction & Elevated track \\
Angle of incidence & $33.9^{\circ}$ \\
Wave & $\mathrm{C}$ \\
Polarization method & $\mathrm{VV}$ \\
Number & 34 \\
Time & $2018 / 06 / 14-2021 / 04 / 11$ \\
\hline
\end{tabular}

\section{Principles of Monitoring and Forecasting Techniques}

\subsection{SBAS-InSAR Technology}

The basic principle of SBAS technique is to take the deformation results obtained by single D-InSAR technique as observations and calculate them by the least squares method to obtain high-precision deformation information on the time series [11-13]. in order to reduce the vectorial coherence phenomenon, generates multi-visual differential interferograms by setting the threshold screening of spatio-temporal baselines when selecting interferometric image pairs, divides these interferograms into several groups according to the baseline conditions, and solves the deformation time series of each group by least squares method, using singular value decomposition (SVD) to unite multiple small baseline sets to obtain the corresponding time series deformation information.

\subsection{BP Prediction Technique}

The BP algorithm learning consists of two processes: signal forward transmission and error back propagation. In the signal forward process, the input information is fed from the input layer, processed through the implicit layer and the result is calculated in the output layer. The information is processed layer by layer, with the value of the neuron in the previous layer only having an effect on the neuron in the next layer. If the output layer result is far from the desired value, it enters the error reverse transfer process. The error results are used to make parameter corrections to the weights and thresholds between the layers of the network, and the above process is repeated until the global error is approximated to a minimum value [14], and the structure of the BP neural network is shown in Figure 2. 


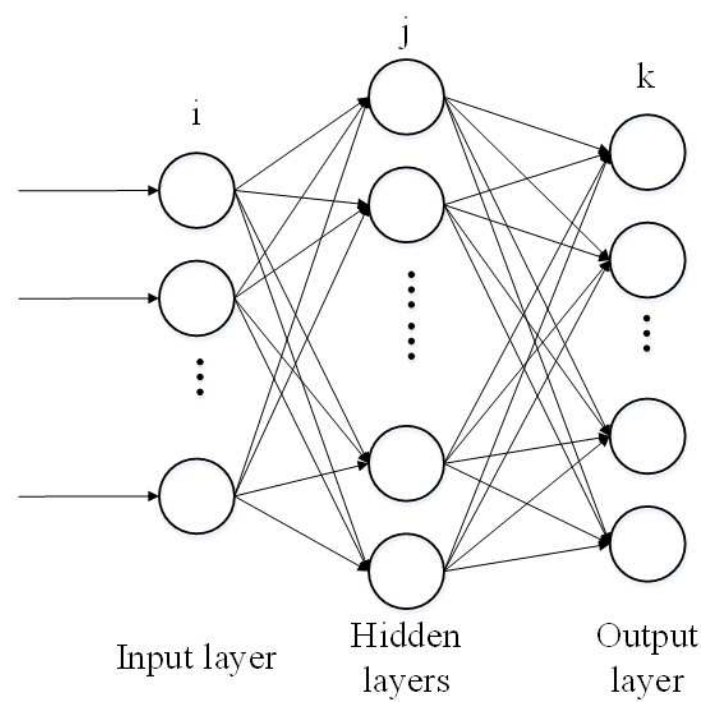

Figure 2. Structure diagram of BP neural network.

\section{Monitoring Technical Data Processing}

SBAS technique is based on ENVI-sarscape software for data processing, before generating interferograms, the original data are imported into the software and pre-processed such as cropping, considering that the three factors of time baseline, spatial baseline and Doppler center will have an impact on the optimal selection of the public master image, SBAS technique takes the image of November 12, 2019 as the public master image, in order to avoid the time and spatial decoherence, after several trials, the maximum critical baseline threshold is set to $3 \%$, the maximum time distance threshold is set to 150 days, and the temporal phase unwinding is done with the Minimum Cost Flow method, Figure 3 shows the SBAS spatio-temporal baseline connection map, after multi-viewing, interferogram generation, filtering, de-leveling effects, orbit refinement and inversion, the interferogram DEM error is estimated, the high and low pass filtering to extract the atmospheric phase, and solve for the line-of-sight deformation information in the study area.

The results solved by SBAS technique were optimized and mapped for settlement rate using ArcGIS software, and finally, the time series analysis was performed using Origin software.

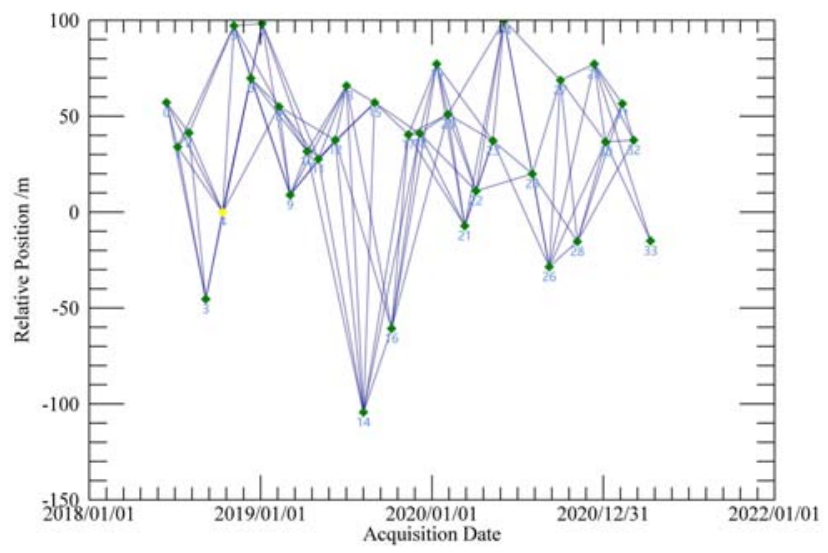

Figure 3. Space-time baseline connection.

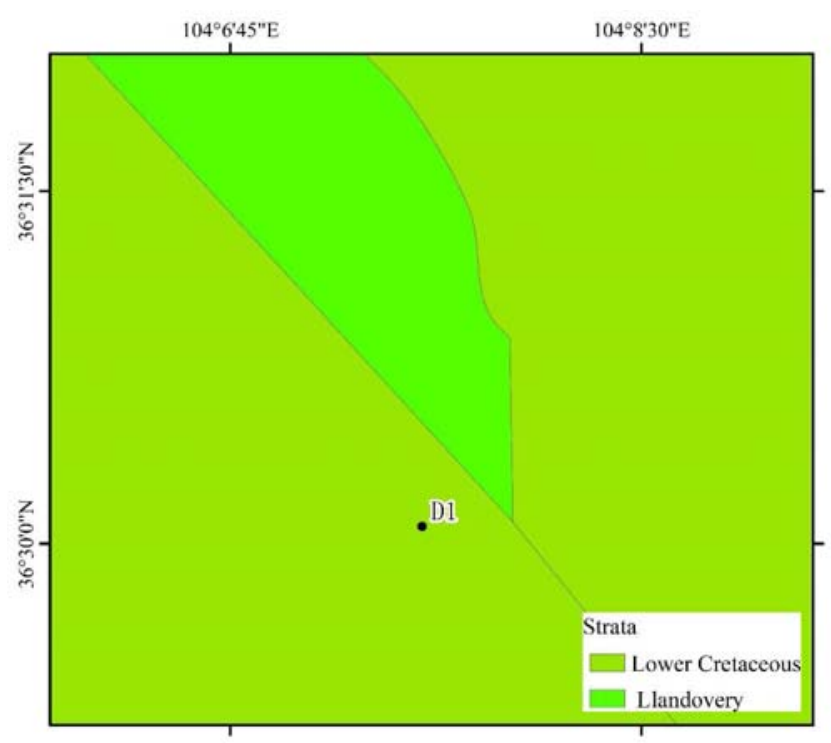

Figure 4. Geological map of Yinxi Industri.
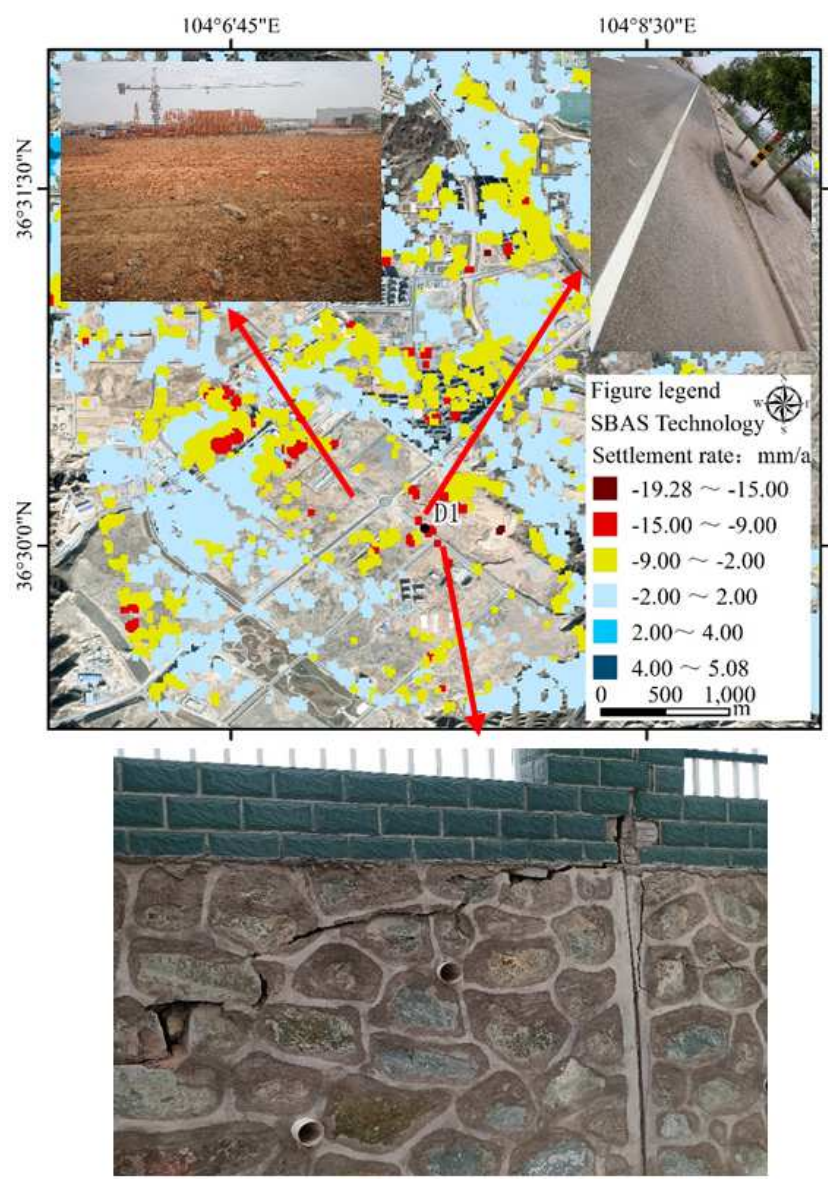

Figure 5. Settlement rate map and site photo map of Yinxi Industrial Park.

\section{Deformation Results and Prediction Analysis}

\subsection{InSAR Results Validation}

Figure 4 shows the geological map of Yinxi Industrial Park, the original stratigraphic lithology of the area is the 
Quaternary wind-deposited loess and chalk sandy mudstone interlayered bedrock rock group, after backfilling by cutting the mountain to make land, the upper foundation soil is uniformly changed into plain fill, the different lithology changes the geotechnical engineering geological conditions, and the leakage of buried underground ducts or pipes may reduce the bearing capacity of the foundation and cause uneven settlement of buildings [15]. At the same time, it may also trigger new geotechnical engineering geological problems, which seriously affect the production and life of local residents. As can be seen in Figure 5, the annual average deformation rates obtained by SBAS technique ranged from $-19.28 \mathrm{~mm}$ to $5.08 \mathrm{~mm}$, and the ground settlement and wall cracking occurred in this area, which is highly consistent with the settlement zone identified by SBAS technique, further proving the reliability of SBAS technique.

In order to study the subsidence pattern in the area, the frequency distribution map of the subsidence rate in the study area was counted, and it can be seen from Figure 6 that the annual deformation rate of the majority of points is between $-15 \mathrm{~mm}$ and $15 \mathrm{~mm}$. The highly coherent subsidence point D1 was selected, and the cumulative subsidence at this point was analyzed in a time series. The cumulative subsidence trend at this point is decreasing, and the cumulative subsidence in 2021 exceeds $30 \mathrm{~mm}$ (Figure 7).

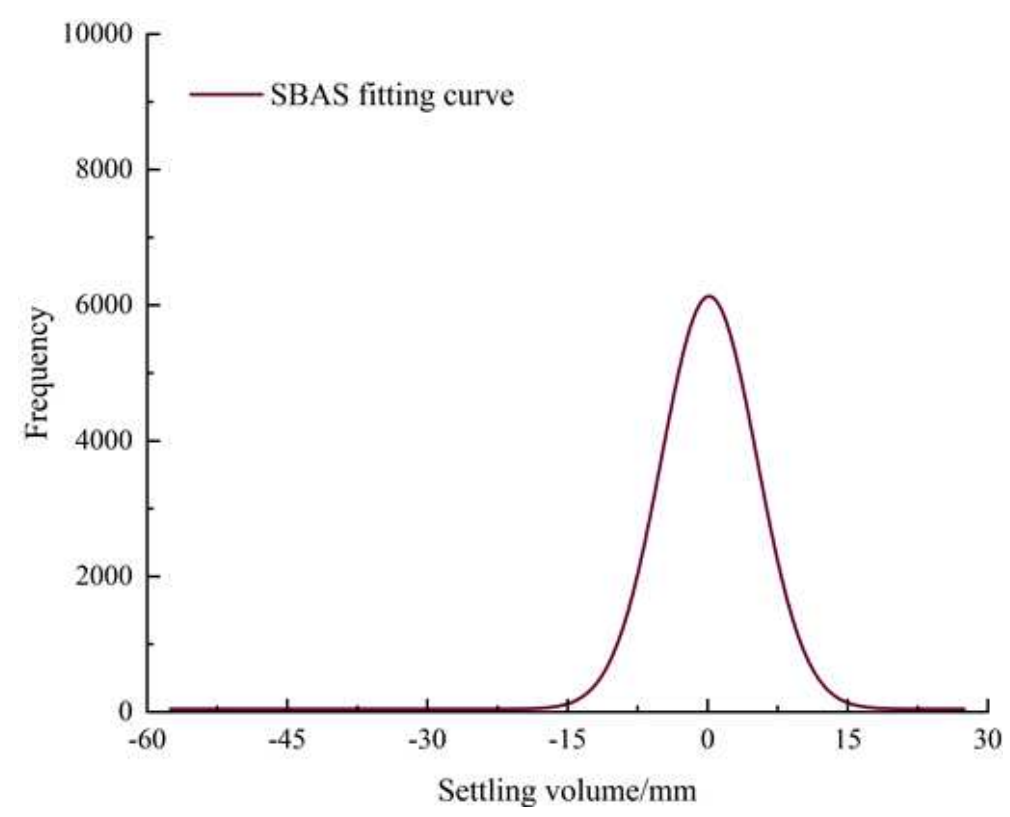

Figure 6. Frequency distribution statistics of cumulative subsidence.

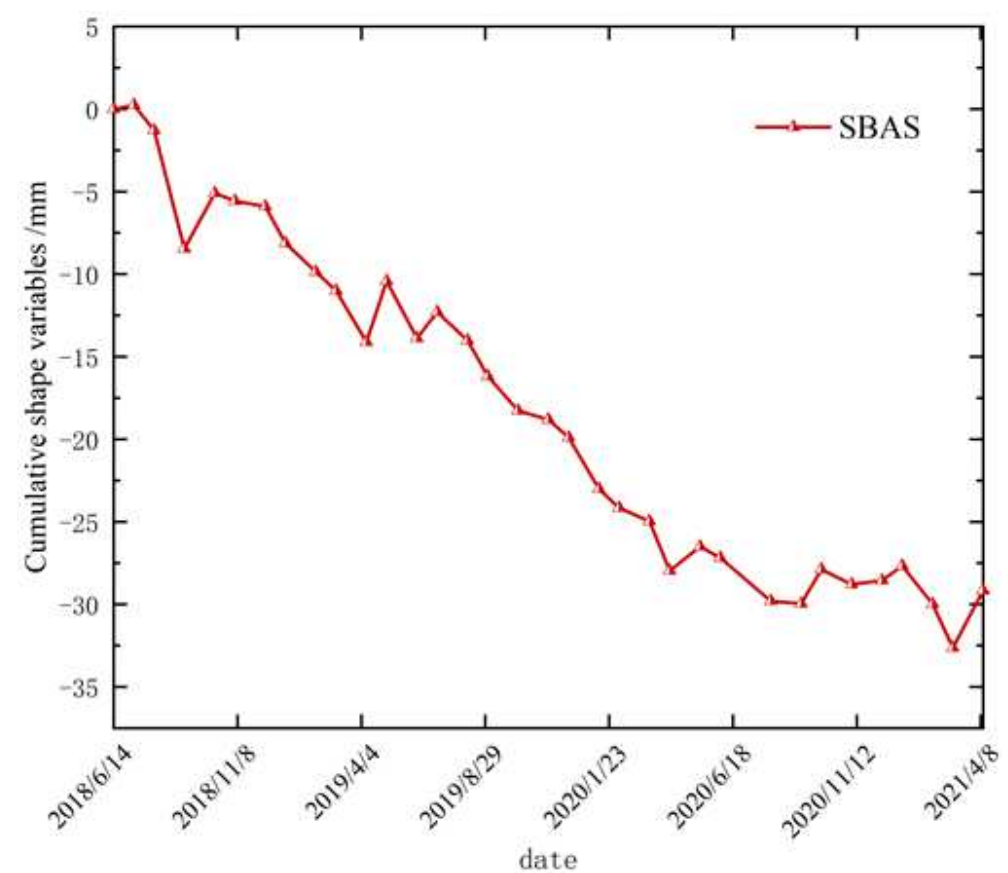

Figure 7. Cumulative settlement at point D1. 


\subsection{BP Neural Network Prediction}

For early identification and prevention of the study area, 1100 PS points were selected from the results obtained with SBAS technique, the first 1000 PS point settlement values were randomly selected as training samples and the last 100 PS point settlement values were used as test samples, the 33 period settlement values from June 14, 2018 to March 6, 2021 were used as the input layer and the settlement values on April 11, 2021 were used as the output layer, the sample data are normalized and prediction analysis is performed based on BP neural network model. Figure 8 shows the actual sedimentation values solved by SBAS technique and the predicted values by neural network. The mean square error is $2.56 \mathrm{~mm}$ and the average relative error is $6.06 \%$, so the prediction accuracy is high. The prediction of the cumulative settlement value at point D1 using BP neural network is shown in Figure 9, and the results show that the settlement value at point D1 will exceed $45 \mathrm{~mm}$ in 2023 , and the cumulative settlement will further increase under the action of external loads such as vehicles because the point is located on the highway.

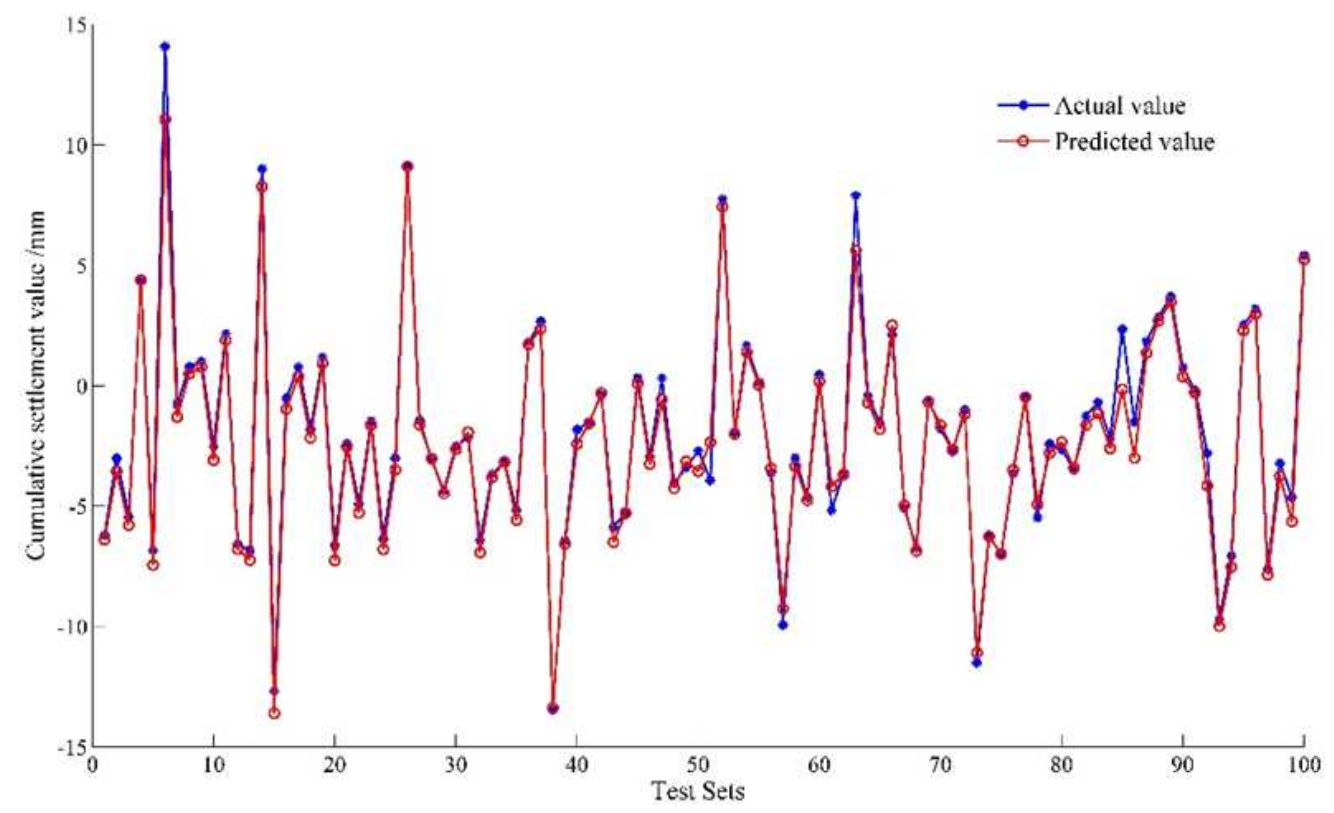

Figure 8. BP neural network prediction results.

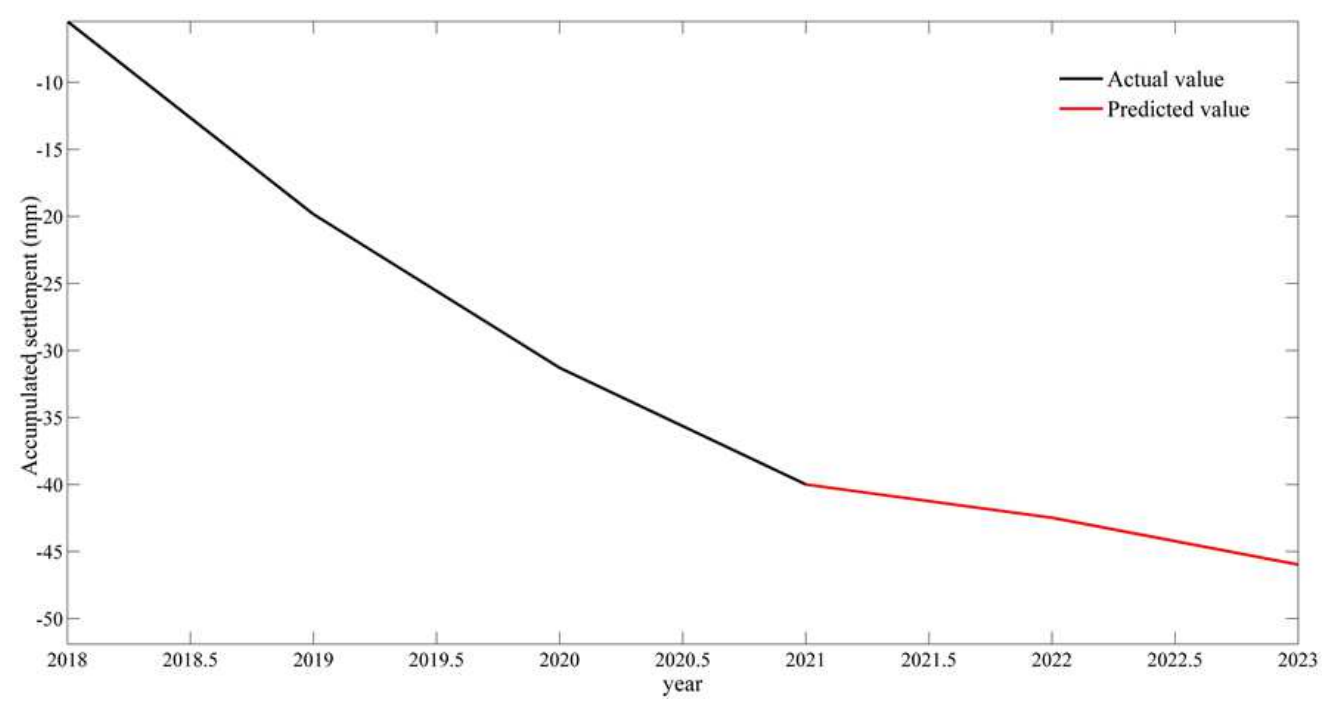

Figure 9. Neural network prediction results.

\section{Conclusion}

In this study, based on 34 scenes of sentinel-1A data, the
SBAS technique was used to obtain the results of subsidence and spatial and temporal distribution in and around the Yinxi Industrial Park in Baiyin with fieldwork verification, combined with geological data and field research for analysis. 
The experimental results show that:

(1) During the monitoring period, the annual average deformation rate of most settlement points in and around Yinxi Industrial Park was between $-15 \mathrm{~mm}$ and $15 \mathrm{~mm}$, especially in the area of roads and buildings, there was more obvious ground settlement, and the annual average settlement rate ranged from $-19.28 \mathrm{~mm}$ to $5.08 \mathrm{~mm}$, and the cumulative settlement amount of D1 points located near the roads exceeded $30 \mathrm{~mm}$, and the field investigation verified that the SBAS technology monitoring results have good reliability.

(2) The spatial distribution of subsidence areas has obvious geological characteristics. The subsidence area is mainly dominated by loess and sandy mudstone, and the upper part of the foundation soil is uniformly changed into plain fill by cutting the mountain to make land, and the stratum is more fragile and easily deformed. Moreover, between 2018 and 2021, large human activities, such as extensive housing reconstruction and rapid urbanization, caused significant ground subsidence in some areas.

(3) The accuracy of the BP neural network prediction results is high, in which the mean square error is 2.56 $\mathrm{mm}$ and the average relative error is $6.06 \%$. The BP neural network predicts that the point D1 will exceed 45 $\mathrm{mm}$ in 2023, and this result is important for the prevention and control of disasters in advance.

(4) Although the current time series detection results of deformation in Yinxi Industrial Park in Baiyin were obtained using Sentinel-1 data and SBAS technology, due to the lack of actual measurement data, the verification of monitoring results was only determined using field research, and future research needs to combine InSAR with traditional monitoring technology to further analyze the mechanism of surface deformation in Yinxi Industrial Park, improve the accuracy and reliability of ground monitoring, and provide scientific and technological support for the regional urbanization process and sustainable urban construction.

\section{Acknowledgements}

On the completion of this thesis, I would like to express my most sincere gratitude and respect to my teachers, Xinghai Dang and Jianyun Zhao, for their guidance. The teachers' perseverance in scientific research, profound knowledge, factual working attitude, generous and modest person, and selfless dedication have always inspired me to keep learning knowledge and pursuing the realm of life. Secondly, I would like to thank the National Natural Science Foundation of China project (51968042) for funding me. Finally, thanks to all the experts and editorial staff for their support.

\section{References}

[1] K Zheng, S. H Yu, Q. S Zhang. Analysis on the causes and prevention methods of land subsidence geological disasters [J]. Surveying and Mapping of Geology and Mineral Resources (2630-4732), 2019, 002 (006): P. 154-155.

[2] Y. Q Zhao. Monitoring the ground subsidence in Xiamen City based on small baseline subset technique [J]. Engineering of Surveying and Mapping, 2020, 29 (2): 46-51.

[3] Z Yang, J Xu. Monitoring the land subsidence in Hexi area of Nanjing by using Sentinel-1A [J]. Bulletin of Surveying and Mapping, 2020, No. 514 (01): 64-68+78.

[4] K. R Dai. Integration of New Generation SAR Data for Extracting the Earth's Surface Topography and Displacement: Methodology and Modelling [D]. Southwest Jiaotong University, 2017.

[5] G. Q Wang. Research on the Monitoring of Geological Hazards on the Bank Reservoir Based on InSAR Technology [J]. China Rural Water and Hydropower, 2020, No. 458 (12): 212-216.

[6] Y Wu. Subsidence monitoring along high speed railway in loess area based on DS-InSAR method [D]. 2019.

[7] F. M Zhao, Y Zhang, X. M Meng, et al. Early identification of geological hazards in the Gaizi valley near the Karakoran Highway based on SBAS-InSAR technology [J]. Hydrogeology \& Engineering Geology, 2020, 47 (01): 142-152.

[8] G. Y Pan, Q. X Tao, Y Chen, et al. Monitoring and analysis of sedimentation in Jiyang mining area of Shandong Province based on SBAS-InSAR [J]. The Chinese Journal of Geological Hazard and Control, 2020, 31 (04): 100-106+120.

[9] Y Zhang. Application of InSAR technology in DEM construction and its accuracy analysis [D]. Kunming University of Science and Technology, 2017.

[10] G. W Jin. Research on Key Processing Techniques for Deriving Accurate DEM from InSAR [D]. PLA Information Engineering University, 2007.

[11] P Berardino, G Fornaro, R Lanari, et al. A New Algorithm for Surface Deformation Monitoring Based on Small Baseline Differential SAR Interferograms [J]. IEEE Transactions on Geoscience \& Remote Sensing, 2002, 40 (11): 2375-2383.

[12] S Usai, R Klees. SAR interferometry on a very long time scale: a study of the interferometric characteristics of man-made features [J]. IEEE Transactions on Geoscience and Remote Sensing, 1999, 37 (4): P. 2118-2123.

[13] S Usai,. A New Approach for Longterm Monitoring of Deformations by Differential SAR Interferometry. delft university press, 2001.

[14] P Zhang, Landslide Susceptibility Based on Time-series InSAR Technology Com bined with Back Propagation [D]. Shandong University of science and technology, 2019.

[15] Z. X Su. Selection of geotechnical investigation method and analysis of bearing capacity for artificial fill under foundation [J]. Building Science, 2021, 37 (07): 139-144. 